\title{
ON TOPOLOGICAL TRANSFORMATION GROUPS ${ }^{1}$
}

\section{LLOYD LININGER}

The purpose of this note is to prove that under certain conditions, if a compact Lie group $G$ acts on a space $M$, then there will exist uncountably many topologically distinct actions of $G$ on $M$.

Montgomery and Yang have recently shown in [3] that $S^{1}$ can act on $S^{7}$ in countably many topologically distinct ways. Their methods are different from the ones employed in this paper. In both cases, the actions are distinguished by the orbit spaces. However, Montgomery and Yang distinguish the orbit spaces, which are in fact differentiable manifolds, by global properties. In this note, the orbit spaces are distinguished by local properties, and hence they are not manifolds.

The notation and terminology are standard. If $G$ acts on $M$, and $x$ is an element of $M$, then $G_{x}$ will denote the isotropy subgroup, $G / G_{x}$ the quotient subgroup, $G(x)$ the orbit of $x, M / G$ the orbit space, and $P_{G}$ the projection map from $M$ to $M / G$. If $A$ is an arc in the interior of a $k$-cell $D$, then $D / A$ will denote the space obtained by identifying $A$ to a point.

TheOREM. Suppose $G$ is a compact Lie group acting continuously on $M$, and there exists an element $x$ in $M$ such that, (1) $x$ is an element of a principal orbit, (2) for any embedding of an arc $A$ in a $k$-cell $D$, there exists a homeomorphism $f$ from $G / G_{x} \times D / A$ onto $G / G_{x} \times D$ whose restriction to $G / G_{x} \times \operatorname{Bd}(D / A)$ is the identity map, and (3) $P_{G}(G(x))$ has a neighborhood $V$ in $M / G$ homeomorphic to $R^{k}, k>2$. Then there exists an uncountable collection of topologically distinct $G$ actions on $M$ with the property that the orbit types of any two elements in the collection are the same.

Proor. Let $V$ be the neighborhood given by the second hypothesis. It follows from the slice theorem of [4], that $P_{G}$ restricted to $P_{G}^{-1}(V)$ is a fiber map, and that $P_{G} \mid P_{G}^{-1}(V)$ is a product fibration.

Suppose $D$ is a closed $k$-cell in $V$, and $A$ is a noncellular arc in the interior of $D$. Let $(M / G) / A$ denote the space obtained from $M / G$ by collapsing $A$ to a point. By hypothesis, there exists a homeomorphism $f$ from $G / G_{x} \times D / A$ onto $G / G_{x} \times D$ whose restriction to $G / G_{x}$ $\times \operatorname{Bd}(D / A)$ is the identity. Let $M^{\prime}$ denote $P_{G}^{-1}(M / G-\operatorname{Int} D)$ $\cup\left(G / G_{x} \times D / A\right)$ with the obvious identification.

Received by the editors September 26, 1967.

1 Work was partially supported by NSF Grant GP-6967. 
Then $G$ acts on $M^{\prime}$, and the corresponding orbit space $M^{\prime} / G$ is homeomorphic to $(M / G) / A$. But $M^{\prime}$ is homeomorphic to

$$
P_{G}^{-1}(M / G-\operatorname{Int} D) \cup f f\left(G / G_{x} \times D / A\right),
$$

which is homeomorphic to $M$. Hence, $G$ acts on $M$ with orbit space $(M / G) / A$, and the orbit type of the new action is the same as the orbit type of the initial action of $G$.

To show there is an uncountable collection of actions satisfying the conclusion of the theorem, one only has to observe that there is an uncountable collection $C$ of topologically distinct closed sets in $D$ such that, for each $B$ in $C$, there exists an action $H$ of $G$ on $M$ such that $H$ agrees with the initial action of $G$ on $P_{G}^{-1}(M / G-B)$, and the set of points at which $P_{H}\left(M-P_{G}^{-1}(M / G-D)\right)$ fails to be euclidean is the set $B$. See [2] for a similar construction.

REMARK 1 . If $G$ is a compact Lie group acting differentiably on a differentiable manifold $M$, then the third hypothesis of the theorem is satisfied for any $x$ in a principal orbit.

COROLlaRY 1. If $G$ is a compact Lie group acting differentiably on a differentiable manifold $M$, and there exists an element $x$ in $M$ such that, (1) $x$ is contained in a principal orbit, and (2) $G / G_{x}$ can be fibered over $S^{1}$, then there exist uncountably many topologically distinct actions of $G$ on $M$.

Proof. This follows easily from a slight extension of the well-known theorem of Andrews and Curtis [1].

REMARK 2. The hypothesis of Corollary 1 is satisfied if $G=S^{1}$.

REMARK 3. The methods of this note do not extend to discrete groups, since the projection map at $x$ is a local homeomorphism.

\section{BIBLIOGRAPHY} 1-7.

1. J. Andrews and M. Curtis, N-space modulo an arc, Ann. of Math. (2) 75 (1962),

2. L. Lininger, Actions on $S^{n}$, (to appear).

3. D. Montgomery and C. Yang, Differentiable actions on homotopy seven spheres. II, (to appear).

4. D. Montgomery and L. Zippin, Topological transformation groups, Interscience, New York, 1955.

UNIVERSITY OF MisSOURI AND

The Institute for Advanced Study 